\title{
Fulgence de Ruspe
}

(saint)

\section{Y. Modéran}

\section{OpenEdition}

Journals

Édition électronique

URL : http://journals.openedition.org/encyclopedieberbere/1975

DOI : 10.4000/encyclopedieberbere.1975

ISSN : 2262-7197

\section{Éditeur}

Peeters Publishers

\section{Édition imprimée}

Date de publication : 1 février 1998

Pagination : 2939-2944

ISBN : 2-85744-994-1

ISSN : 1015-7344

\section{Référence électronique}

Y. Modéran, «Fulgence de Ruspe », Encyclopédie berbère [En ligne], 19| 1998, document F52, mis en ligne le 01 juin 2011, consulté le 24 septembre 2020. URL : http://journals.openedition.org/ encyclopedieberbere/1975; DOI : https://doi.org/10.4000/encyclopedieberbere.1975

Ce document a été généré automatiquement le 24 septembre 2020.

(c) Tous droits réservés 


\title{
Fulgence de Ruspe
}

(saint)

\author{
Y. Modéran
}

1 «Le plus grand théologien et le plus saint évêque de son temps " selon Bossuet, Fulgence est aussi la figure la plus marquante du catholicisme africain à l'époque de la domination vandale, et c'est surtout en tant qu'Africain qu'il sera ici présenté. Fait exceptionnel pour cette époque, sa vie nous est relativement bien connue grâce à une biographie, la Vita Fulgentii, écrite peu après sa mort par un de ses proches (peut-être Ferrand de Carthage), et grâce à ses nombreuses œuvres conservées (traités, sermons, lettres).

\section{Les origines et la jeunesse (468-vers 493)}

2 Fulgence naquit à Thélepte, dans le sud-ouest de la Byzacène, en 468. Selon la Vita (I), par son grand-père Gordianus et son père Claudius, il appartenait à une famille "noble" de "sénateurs carthaginois" (parentes habuit ex numero Carthaginensium senatorum). L'expression peut simplement évoquer le sénat de Carthage, c'est-à-dire la curie municipale, qui se qualifiait souvent elle-même de "très splendide assemblée ". Mais elle peut aussi signifier que Gordianus et sa famille étaient de véritables clarissimes, membres de l'ordre sénatorial romain. Plusieurs auteurs ont privilégié dans les dernières années cette interprétation. Ils ont ainsi mis en valeur la dignité incontestable de nombre de correspondants de Fulgence au vi siècle, comme l'ancien consul (505) Theodorus, ou les nobles Galla et Proba, "petites filles de consuls de Rome" : leurs liens avec l'évêque de Ruspe s'expliqueraient par des relations anciennes entre grandes familles de l'ordre sénatorial. En fait, l'argument n'est pas irréfutable car, comme nous le verrons, Fulgence devint après 508 une personnalité éminente de l'Église en Occident, et il n'y a rien d'étonnant à ce que, comme jadis saint Jérôme ou saint Augustin, les plus grands nobles l'aient alors consulté.

On a également mis en valeur la mention vir clarissimus qui accompagne sur certains manuscrits le nom de Fabius Claudius Gordianus Fulgentius, auteur d'une curieuse histoire chrétienne du monde, le De aetatibus mundi et hominum, qui pourrait être 
l'évêque de Ruspe. Ce second Fulgence vivait probablement à la fin $d u v^{e}$ ou au $v^{e}$ siècle, était Africain et portait des noms indiscutablement proches de notre personnage (Claudius, nom du père de Fulgence, Gordianus, nom de son grand-père). Mais le silence total de la Vita sur cette œuvre, très différente par son orientation des écrits du saint évêque, son style et ses particularités littéraires ont conduit beaucoup d'historiens à refuser l'assimilation et à distinguer deux Fulgence, voire même trois puisqu'on connaît également un Fabius Planciades Fulgentius, auteur de plusieurs opuscules mythographiques, qui semble aussi avoir été un Africain contemporain de l'époque vandale. En fait, cette question des rapports entre les différents Fulgence reste une des plus confuses de l'histoire littéraire des $\mathrm{v}^{\mathrm{e}}$ et $\mathrm{vI}^{\mathrm{e}}$ siècles, et il parait bien difficile d'en faire état pour préciser le milieu social du futur évêque de Ruspe.

Quelle que soit l'hypothèse retenue, il semble que l'on ne puisse cependant hésiter sur l'origine aristocratique de la famille. Curiale de Carthage ou notable africain honoré du clarissimat sans résider à Rome, comme beaucoup d'autres riches provinciaux du BasEmpire, Gordianus appartenait à l'élite de la société africaine romanisée. A ce titre, il fut donc logiquement parmi les premières victimes de la conquête vandale: en 439, ayant subi sur décision du roi Genséric la confiscation de tous ses biens, il s'exila avec tous les siens en Italie. Mais le pouvoir vandale eut vite besoin pour s'affirmer de la collaboration des élites romaines d'Afrique. Genséric modifia donc sa politique et par la suite, après la mort de Gordianus et avant 468 , ses fils, dont Claudius, furent autorisés à rentrer en Afrique. Ils purent même bénéficier d'une mesure de restitution partielle de leurs propriétés, limitée toutefois à la Byzacène. Cette restriction s'explique probablement par la différence de statut entre les provinces du royaume : la Byzacène formait une partie du territoire réservé au roi, et il pouvait donc y décider de restitutions sans léser ses guerriers. La chose était par contre très difficile en Proconsulaire, où ces derniers avaient été massivement lotis. Lorsque Fulgence naquit, sa famille avait ainsi retrouvé l'essentiel de son statut social, et toute la jeunesse du futur évêque illustre de fait son appartenance à l'élite traditionnelle. Ayant perdu très tôt son père, il fut élevé par sa mère Mariana, et reçut sous sa direction une éducation à la fois chrétienne et classique, qui fit une grande place à l'apprentissage du grec. Devenu adulte, Fulgence prit en mains la gestion des domaines familiaux. Il entra alors probablement à la curie de Thélepte, et fut nommé selon la Vita (I) "procurateur", ce qui pourrait signifier ici qu'il assuma au nom du conseil municipal la charge de perception des impôts dûs par les habitants de la cité à l'État vandale. A cette époque, au début des années 490, Fulgence était ainsi devenu un bon exemple de la collaboration effective qui s'était établie entre les élites laïques africaines et le pouvoir vandale.

\section{Un moine instable (vers 493-508)}

5 Vers 493, alors que la persécution anticatholique poursuivie depuis Genséric s'atténuait fortement sous l'égide du roi Gunthamund, Fulgence, mu par un désir d'ascétisme qui n'allait plus le quitter, décida de renoncer au monde et de rejoindre la communauté monastique de l'évêque Faustus, établie non loin de Thélepte. Il ne put cependant demeurer longtemps dans ce premier monastère: vers la fin 495, un changement d'attitude de Gunthamund et un retour de la persécution contraignirent en effet Faustus et ses moines à se disperser et à se cacher. Fulgence gagna alors un second 
établissement, assez proche, dirigé par un abbé nommé Félix. Il n'y demeura guère plus d'un an ou deux car, vers 497, une incursion de Maures hostiles dans le sud-ouest de la Byzacène, prolongement du soulèvement commencé dans l'Aurès vers 484 , l'obligea à fuir avec Félix vers des régions plus calmes, près de Sicca en Proconsulaire. A peine arrivés, les deux moines ne trouvèrent cependant pas la paix espérée : capturés et battus sur l'ordre d'un prêtre arien désavoué ensuite par son évêque, Fulgence et Félix repartirent vers le sud pour s'installer finalement à Mididi, où ils fondèrent un nouveau monastère (vers 497-498). A l'abri désormais des incursions maures et d'une persécution vandale qui apparemment ne frappait pas les moines, Fulgence ne sut pour autant demeurer longtemps en ce lieu. Toujours désireux de plus d'ascétisme, il décida au début de 499 de gagner l'Égypte pour partager l'existence des ermites du désert. Après une sortie d'Afrique qui semble-t-il était alors tout à fait légale, il fit escale en Sicile où l'évêque de Syracuse réussit à le persuader de renoncer à son projet. La fuite vers le désert se transforma ainsi en un pèlerinage à Rome, au début de l'an 500 : Fulgence visita le tombeau des apôtres, assista à l'entrée triomphale du roi ostrogoth Théodoric, puis rentra en Afrique. Il ne se résigna pourtant pas à demeurer à Mididi, et préféra fonder, vers 501, un nouveau monastère (le quatrième) en Byzacène, probablement à proximité relative de la cité côtière de Ruspe. Tout indique que dès ce moment sa réputation était déjà considérable dans la province puisque, selon la Vita (X), nombreux étaient alors les notables (nobiles viri) qui le connaissaient et l'entouraient. On ignore cependant si ce sont déjà ses écrits, ou plutôt sa réputation d'ascète qui lui valaient cette célébrité.

Les voyages de saint Fulgence en Afrique (carte d'Y. Modéran).

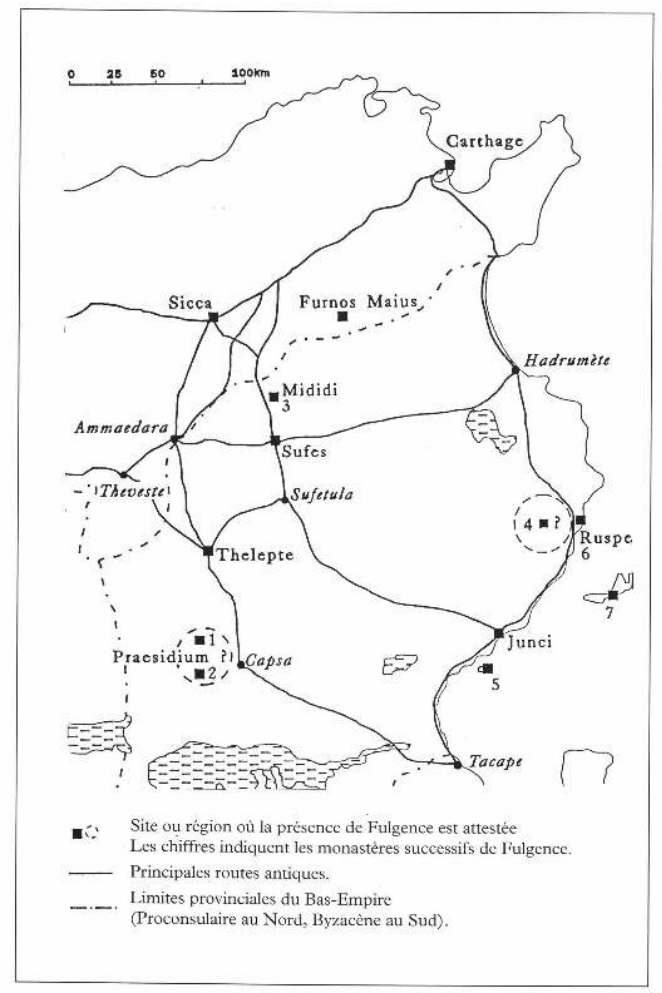

6 Malgré celle-ci, et même si son monastère devint très vite un établissement important, Fulgence ne tarda pas à manifester une nouvelle fois une insatisfaction qui tendait à devenir chronique. Vers 505-506 (?), désireux de toujours plus d'ascétisme, il s'enfuit 
secrètement et rejoignit une communauté marginale établie au large de Macomades Junci, sur un banc rocheux isolé des îles Kneiss, récemment identifié avec une quasicertitude. Le scandale de cette fuite fut grand et c'est l'évêque Faustus lui-même, son ancien supérieur, qui obtint finalement sous la menace de l'excommunication le retour de l'incorrigible ascète, dont on crut désormais s'assurer de la stabilité en lui conférant la prêtrise (vers 506-507 ?) puis le siège épiscopal de Ruspe (508).

\section{L'évêque de la résistance (508-523)}

7 Les pérégrinations de Fulgence reprirent cependant très vite, mais cette fois pour des raisons indépendantes de sa volonté. Peu après son ordination, et après la construction d'un nouveau monastère à Ruspe (le sixième), il fut en effet arrêté et exilé en Sardaigne, près de l'actuelle Cagliari. Cette expulsion était la conséquence d'une mesure générale décidée par le roi Thrasamund, furieux d'avoir constaté en 508 de nombreuses ordinations épiscopales malgré une interdiction formelle remontant au moins au début des années 480 et qui constituait l'élément central de la politique anticatholique du pouvoir vandale. Fulgence dut donc s'embarquer pour la Sardaigne avec une soixantaine d'autres évêques, tandis qu'un nombre à peu près égal de ses collègues était dispersé en Afrique même. Cet exil, qui allait durer quinze ans, devait marquer le tournant probablement le plus décisif de sa carrière. Certes, aussitôt sur place, il ne put s'empêcher de fonder un nouveau monastère où il consolida, tant auprès de ses confrères que de la noblesse sarde, sa réputation de sainteté. Mais c'est surtout alors par sa science, sa sûreté et son éloquence théologiques qu'il acquit une autorité déterminante. Tout indique en effet que c'est pendant ce long exil qu'il composa ses grands traités anti-ariens sur La Trinité, qui allaient faire sa réputation de docteur et d'héritier de saint Augustin, et finalement devaient attirer l'attention de Thrasamund. A une date inconnue, vers 515-517 (?), ce roi érudit, arien convaincu, soucieux de maîtriser un théologien dont la réputation devenait dangereuse, invita en effet Fulgence à venir débattre avec lui à Carthage. Mais, contre toute attente, le retour temporaire de l'évêque de Ruspe ne fit qu'accentuer son influence. Affrontant avec succès le roi et ses conseillers, il multiplia aussi les contacts avec les catholiques africains et surtout rédigea en quelques mois une impressionnante série d'ouvrages, qui provoquèrent finalement une nouvelle expulsion (vers 518-519 ?). Réinstallé à Cagliari, Fulgence fut alors probablement en Occident l'évêque le plus célèbre de son temps. Il réunissait en effet en sa personne trois vertus exceptionnelles : celle d'un modèle de sainteté par son ascétisme et sa vie monastique, celle d'un théologien dont l'orthodoxie augustinienne paraissait si sûre qu'on le consultait même d'Orient (en particulier sur les résurgences gauloises du pélagianisme), et celle d'un héros de la résistance catholique à la persécution vandale.

\section{Les dernières années : la gloire et l'ascétisme (523-533)}

8 On ne peut dès lors s'étonner de l'enthousiasme extraordinaire qui salua le retour définitif de Fulgence à Carthage, lorsqu'en 523 le nouveau roi Hildéric rappela tous les exilés et établit pour la première fois une véritable tolérance. Cependant, salué par tous ses collèges et fêté par les communautés catholiques de tout le pays, Fulgence montra 
alors immédiatement qu'il n'entendait en rien changer son style de vie. A la fois évêque et moine à Ruspe, il ne chercha certes plus à fuir ses responsabilités : il participa ainsi à des conciles à Sufes et à Junci en 523, et on le vit administrer avec attention son diocèse. Mais en même temps il ne cessa aussi de manifester sa préférence pour son monastère, où il pouvait continuer à composer de nouveaux ouvrages et où surtout il pouvait satisfaire son aspiration à l'ascétisme et au recueillement. Fidèle à ce qui était en fait son véritable idéal, et apparemment indifférent aux troubles provoqués en 529 par un soulèvement berbère puis en 530 par le coup d'État du roi Gélimer, il en vint même finalement une nouvelle fois, vers 531-532, à s'enfuir secrètement pour rejoindre un établissement monastique isolé, établi sur une des îles Kerkenna. Et ce n'est que sous la contrainte d'appels répétés de sa communauté, comme trente ans auparavant, qu'il rentra à Ruspe où il mourut peu de temps après, le soir du I $\mathrm{I}^{\mathrm{er}}$ janvier 533. Quelques mois plus tard, Bélisaire débarquait à Caput Vada, à moins de vingt kilomètres de là, et l'empire d'Orient, en rétablissant l'autorité romaine en Afrique, assurait aussi le triomphe définitif de l'église catholique sur l'arianisme vandale.

\section{Fulgence et l'Afrique}

Nul doute que l'engagement obstiné de Fulgence pendant quarante ans au service de l'orthodoxie et contre toutes les persécutions n'ait largement contribué à cette victoire finale. Mais s'il est ainsi un témoin exceptionnel de la vigueur des luttes religieuses qui divisèrent son pays sous les successeurs de Genséric, l'évêque de Ruspe illustre également bien par sa vie les réalités contradictoires et les ambiguités de l'Afrique vandale. Celle-ci apparaît en effet tout au long de cette carrière mouvementée comme un monde resté profondément romain dans ses structures.

Le pays dans lequel évolue Fulgence a conservé ses cités, ses institutions et ses élites municipales, son organisation administrative, et ses routes, que le moine instable ne cesse de sillonner. Tout ici semble s'inscrire dans une continuité avec le Bas-Empire, au point que $\mathrm{Ch}$. Saumagne crut jadis pouvoir conclure de cette vie à la prédominance d'une "paix vandale". Ce serait pourtant oublier que l'Afrique fut aussi, à cette même époque, le théâtre d'une formidable renaissance berbère, illustrée en particulier par les grands soulèvements de l'Aurès vers 484 et surtout de Byzacène à partir de 529 . Ce phénomène, qui n'exclut pas d'ailleurs la poursuite du mouvement de christianisation des tribus entamé au siècle précédent, a nécessairement marqué la vie de Fulgence. Mais, plus encore que son modèle Augustin, l'évêque de Ruspe semble avoir totalement ignoré ses voisins berbères. Tout au plus pourrait-on relever, si on admet l'équivalence des deux auteurs, une indication dans le De cetatibus mundi et hominum (éd. Helm, Leipzig, 1898, p. 131) sur le nombre de lettres que comportait l'alphabet libyque. Mais pour le reste, jamais Fulgence ne parait avoir évoqué les Maures dans ses œuvres. Né au sein de l'aristocratie romano-africaine, il ne s'éloigna en fait jamais de celle-ci, qui d'ailleurs le protégea souvent. De l'évêque arien de la région de Sicca, ami de sa famille, aux nobles citoyens de Byzacène toujours prêts à lui offrir des terrains pour ses monastères, les moments significatifs de ces relations continûment entretenues sont innombrables. En revanche, les seules occasions où son destin le met en rapport avec les Maures reflètent surtout une complète ignorance mutuelle. Sans revenir sur la fuite de son second monastère en 496-497 à la suite d'une "incursion de barbares", il n'est de meilleur symbole de cette coupure profonde que ce qu'il advint de l'église de Ruspe peu 
après la mort de son saint évêque, en janvier 533 : la gens inimica Maurorum, dit la Vita, saccagea alors le territoire de la cité, « dévastant tout par les incendies, les meurtres et les pillages, égorgeant dans les murs même de l'église ceux qu'elle put trouver ». De toute évidence, l'héritier du "sénateur" Gordianus et correspondant du consul Théodoras ne s'était guère soucié à la fin de sa vie de rencontrer les grands chefs berbères du moment, Antalas et Cusina. "Destiné à vivre parmi les Africains » comme disait sa mère Mariana (victurus inter Afros), il semble n'avoir surtout connu de ces derniers que les seules populations romanisées.

\section{BIBLIOGRAPHIE}

Vie de saint Fulgence de Ruspe, édition et traduction française par le Père G.-G. Lapeyre, Paris, 1929. Sancti Fulgentii episcopi Ruspensis opera, édi. J. Fraipont, Corpus Christianorum, s. Latina, t. XCI-XCI A, Turnhout 1968.

Lapeyre G.-G., Saint Fulgence de Ruspe. Un évêque africain sous la domination vandale, Paris, 1929. SAUMAGNE Ch., “La paix vandale”, Revue Tunisienne, 1930, p. 167-184. courtois C, Les Vandales et l'Afrique, Paris, 1955.

LANGLOIS P., "Les œuvres de Fulgence le Mythographe et le problème des deux Fulgence", Jahrbuch für Antike und Christentum, 7, 1964, p. 94-105.

STEVENS S., “The Circle of Bishop Fulgentius”, Traditio, 28, 1982, p. 327-341.

TROUSSET P., SLIM H., PASKOFF R., OUESLATI A., "Les îles Kneiss et le monastère de Fulgence de Ruspe”, Antiquités Africaines, t. 28, 1992, p. 223-247.

NAF B., "Fulgentius von Ruspe, Caesarius von Arles und die Versammlungen der römischen Senatoren", KLIO, 74, 1992, p. 431-446.

MODERAN Y., "La chronologie de la vie de saint Fulgence de Ruspe et ses incidences sur l'histoire de l'Afrique vandale", M.E.F.R.A., t. 105, 1993, p. 135-188.

\section{INDEX}

Mots-clés : Antiquité, Christianisme, Tunisie 